\title{
Neuroprotective effects of erythropoietin on Alzheimer's dementia model in rats
}

\author{
Betul Cevik ${ }^{1, A-F}$, Volkan Solmaz ${ }^{2, A-C}$, Gurkan Yigitturk ${ }^{3, B, C}$, Turker Cavusoğlü ${ }^{3, B, C}$, Gonul Peker ${ }^{4, B, C}$, Oytun Erbas ${ }^{5, A-F}$ \\ ${ }^{1}$ Department of Neurology, Faculty of Medicine, Gaziosmanpasa University, Tokat, Turkey \\ 2 Department of Neurology, Turhal State Hospital, Tokat, Turkey \\ ${ }^{3}$ Department of Histology and Embryology, Ege University, Faculty of Medicine, Izmir, Turkey \\ ${ }^{4}$ Department of Physiology, Ege University, Faculty of Medicine, Izmir, Turkey \\ ${ }^{5}$ Department of Physiology, Istanbul Bilim University, Faculty of Medicine, Istanbul, Turkey \\ A - Research concept and design, B - Collection and/or assembly of data, C - Data analysis and interpretation, \\ $D$ - Writing the article, $E$ - Critical revision of the article, $F$ - Final approval of article
}

\section{Address for correspondence}

Betul Cevik

E-mail: cevikbetul78@hotmail.com

\section{Funding sources}

none declared

Conflict of interest

none declared

Received on August 4, 2015

Revised on May 14, 2015

Accepted on December 18, 2015

\section{Abstract}

Background. Although Alzheimer's disease (AD) is the most common age-related neurodegenerative disease and characterized by memory impairment, only symptomatic treatments are available.

Objectives. Because recombinant human erythropoietin (rhEP0) has various neuroprotective effects and improves cognitive function in animal models of neurodegenerative disorders, we investigated the therapeutic effects of rhEPO in an intracerebroventricular (ICV)-streptozotocin (STZ) animal model of sporadic-AD.

Material and methods. A total of 24 Sprague-Dawley adult rats were divided into 4 groups of naive control $(n=6)$, sham-operated $(n=6), I C V-S T Z+$ saline $(n=6)$ and ICV-STZ + rhEPO $(n=6)$. Twelve rats with Alzheimer's disease, induced by STZ injection (3 mg/kg) into both lateral ventricles using a stereotaxic frame (bilaterally ICV-STZ), were divided into 2 groups 5 days after the STZ injection: one treated with rhEPO 5000 (IU/kg/day, i.p.) and the other with $0.9 \% \mathrm{NaCl}(1 \mathrm{~mL} / \mathrm{kg} / \mathrm{day}$, i.p.) for 2 weeks. The sham-operated rats received bilaterally $I \mathrm{CV}-0.9 \% \mathrm{NaCl}$. No surgical operation or treatment was given to the naive-control animals. On day 20, a passive avoidance learning (PAL) test was used followed by sacrification and removal of the brain tissue in all animals. Brain TNF- $a$ and ChAT levels were determined, and neurons in the hippocampal CA1 and CA3 regions were counted by Cresyl violet staining.

Results. ICV-STZ was found to significantly shorten the latency time on the PAL, increase brain TNF-a level, and decrease brain ChAT activity and the number of neurons in the hippocampal CA1 and CA3 regions. On the other hand, rhEPO significantly attenuated all these detrimental effects induced by STZ.

Conclusions. RhEPO treatment significantly prevented the ICV-STZ-induced memory deficit by attenuating the hippocampal neuronal loss, neuroinflammation and cholinergic deficit in rats. This result suggests that rhEPO may be beneficial for treating AD.

Key words: Alzheimer's disease, erythropoietin, streptozotocin, neuroprotection, hippocampus

DOI

DOI: $10.17219 /$ acem/61044

\section{Copyright}

Copyright by Author(s)

This is an article distributed under the terms of the

Creative Commons Attribution Non-Commercial License

(http://creativecommons.org/licenses/by-nc-nd/4.0/) 
Alzheimer's disease (AD) is the most prevalent age-related neurodegenerative disorder, which leads to progressive development of cognitive deficits with severe learning and memory loss, personality and behavioral changes and eventually to morbidity and death. A number of different familial genetic mutations have been linked to onset, however, the vast majority of $\mathrm{AD}$ is considered sporadic with the exact cause(s) unknown. ${ }^{1,2}$ The neuropathological hallmarks of AD include excessive accumulation of extracellular amyloid beta $(\mathrm{A} \beta)$ plaques and intraneuronal neurofibrillary tangles, along with dystrophic neurites, loss of neurons and synapses, and gliosis in discrete regions of the basal forebrain, hippocampus, and association cortices. Insulin resistance, oxidative stress, glutamate excitotoxicity, mitochondrial dysfunction and neuroinflammation are also among the major pathophysiological features of $\mathrm{AD} .^{3,4}$ Because $\mathrm{AD}$ prevalence is increasing all over the world, the disease represents a major medicosocial problem. However, because current treatments are only symptomatic, effective therapies are needed for the prevention and treatment of AD. ${ }^{5}$

Erythropoietin (EPO) is a growth hormone and cytokine originally described as stimulating erythropoiesis. It is produced mainly by the fetal liver and the adult kidney in response to hypoxia. EPO and its receptors (EPOR) are also found in the nervous system of mammals, and are essential for neurodevelopment, adult neurogenesis and neuroprotection. ${ }^{6,7}$ Recombinant human EPO (rhEPO) has been synthetically produced by using recombinant DNA technology. Immunologically, rhEPO is similar to endogenous EPO and exhibits full biological activity and does not show a species boundary. ${ }^{8}$ It is widely used for the treatment of anemic patients due to chronic renal failure, chemotherapy and prematurity and found to be well-tolerated and safe. Interestingly, in addition to the anti-anemic effect, these patients have also showed improved cognitive abilities. ${ }^{9,10}$ Systemically administered high-dose rhEpo ( $\geq 500 \mathrm{IU} / \mathrm{kg}$ body weight) can cross both the injured and intact blood-brain barrier (BBB) and accumulate in the brain parenchyma in therapeutically effective concentrations. ${ }^{7-9}$ It reduces brain damage and improves the neurological symptoms and cognition in animal models of neurodegenerative diseases, such as focal/global cerebral ischemia, traumatic brain injury, autoimmune encephalitis, epilepsy, septic encephalopathy and even Alzheimer's disease, by inhibiting apoptosis, reducing oxidative stress and inflammation, promoting the angiogenesis and neurogenesis, and maintaining BBB integrity. ${ }^{7-11}$ These results suggest that rhEPO may represent an optimal candidate for use in AD studies.

Intracerebroventricular (ICV) injection of streptozotocin (STZ) causes inflammation, impairment of brain metabolism, cholinergic deficits, neuronal loss and other Alzheimer-like alterations resulting in cognitive dysfunction, as a valid model of sporadic-AD. ${ }^{12,13}$ In light of the results of the above-mentioned previous studies, we in- vestigated the effects of intraperitoneal (i.p.) administration of rhEPO on memory deficit, neuroinflammation, and biochemical and histological alterations in ICV-STZ induced rats.

\section{Material and methods}

\section{Animals}

In this study, 24 Sprague-Dawley adult male rats weighing 200-220 g each were used. The animals were fed ad libitum and housed in pairs in temperature controlled steel cages $\left(22 \pm 2^{\circ} \mathrm{C}\right)$ with $12 \mathrm{~h}$ light and dark cycles. The study was approved by the Committee for Animal Research of Cumhuriyet University. The animal study strictly adhered to the animal experiment guidelines of the Committee for Animal Care.

\section{Experimental protocol}

The animals were randomly divided into 3 groups: stereotaxic infusion of STZ (STZ group, $\mathrm{n}=12$ ) or $0.9 \%$ $\mathrm{NaCl}$ (sham, $\mathrm{n}=6$ ) and a healthy control group. Under general anesthesia induced by i.p. injection of a mixture of ketamine hydrochloride $\left(80 \mathrm{mg} / \mathrm{kg}\right.$, Alfamine ${ }^{\circledR}$, Ege Vet, Alfasan International B.V., Holland) and xylazine hydrochloride $\left(4 \mathrm{mg} / \mathrm{kg}\right.$, Alfazyne ${ }^{\circledR}$, Ege Vet, Alfasan International B.V., Holland), the animals were placed in a stereotaxic frame. STZ $(3 \mathrm{mg} / \mathrm{kg} \mathrm{STZ}$ in $0.9 \% \mathrm{NaCl}$, $\mathrm{n}=12)$ was infused into the left $(2.5 \mu \mathrm{L})$ and right lateral ventricle $(2.5 \mu \mathrm{L})(\mathrm{ICV})(\mathrm{AP}=-0.8 \mathrm{~mm}, \mathrm{~L}= \pm 1.6 \mathrm{~mm}$, DV $=-4.2 \mathrm{~mm}$ ) with a 28 -gauge Hamilton syringe. ${ }^{14}$ The sham-operated rats received the same amount of vehicle $(0.9 \% \mathrm{NaCl})$ into the left and right lateral ventricles. The needle was left in place for an additional $2 \mathrm{~min}$ for complete diffusion of the drug. After the surgery, the rats were monitored daily for behavior and health conditions. The control rats did not undergo any surgical operation.

\section{Erythropoietin treatment}

The ICV-STZ rats were randomly divided into 2 groups 5 days after the STZ injection; Group $1(n=6)$ received saline $(0.9 \% \mathrm{NaCl})(1 \mathrm{~mL} / \mathrm{kg} /$ day, i.p. $)$ and Group $2(\mathrm{n}=6)$ received rhEPO $5000 \mathrm{IU} / \mathrm{kg} /$ day (Eprex ${ }^{\circledR}$, Santa Farma, Istanbul, Turkey) intraperitoneally (i.p.) for 2 weeks.

\section{Passive avoidance task}

After two weeks of the treatment period, a passive avoidance task evaluating the learning and memory were performed in the study and control groups. Passive avoidance learning (PAL) is a trial of a fear-motivated avoidance task in which a rat learns to refrain from stepping 
through a door that would seem apparently safer but actually leads to a dark compartment. The PAL box in a size of $20 \times 20 \times 20 \mathrm{~cm}$ with dark and light sections was used. This PAL box has a grid system that performs electric shocks within the dark compartment. Normally, rats prefer to enter the dark compartment. After $10 \mathrm{~s}$ of a habituation period in the light section area, the guillotine door separating the light and dark chambers was opened. When the rat passed to the dark compartment, the door between the compartments was closed. A $1.5 \mathrm{~mA}$ electric shock was applied for $3 \mathrm{~s}$, and the rat was removed from the dark chamber and returned to its home cage. After $24 \mathrm{~h}$, the rats were re-evaluated in the same manner. The time (latency) to switch from the light compartment to the dark compartment of the rats was recorded, but at this time a shock was not delivered. The latency time was recorded up to a maximum of $300 \mathrm{~s}$. The latency to refrain from crossing into the punishing compartment serves as an index of the ability to avoid and allows memory to be assessed. After testing, the animals were weighed and euthanized. The brains were removed for histopathological and biochemical evaluations.

\section{Determination of choline acetyltransferase (ChAT) activity in brain tissue}

The frozen brain tissue was homogenized and a $10 \%$ cerebral homogenate $(1: 10, \mathrm{w} / \mathrm{v})$ in cold saline was centrifuged at $3500 \times \mathrm{g}$ for $10 \mathrm{~min}$ at $4^{\circ} \mathrm{C}$. The supernatant was used to measure the activity of ChAT by measuring the optical density (OD) at a wavelength of $324 \mathrm{~nm}$. The ChAT activity was expressed as $\mathrm{U} / \mathrm{g}$ protein.

\section{Detection of tumor necrosis factor-alpha (TNF-a) levels in brain tissue}

The frozen brain tissue was homogenized in $1 \mathrm{~mL}$ of buffer containing $1 \mathrm{mmol} / \mathrm{L}$ of PMSF, $1 \mathrm{mg} / \mathrm{L}$ of pepstatin A, $1 \mathrm{mg} / \mathrm{L}$ of aprotinin, and $1 \mathrm{mg} / \mathrm{L}$ of leupeptin in a PBS solution ( $\mathrm{pH} 7.2)$. The mixture was centrifuged at $12,000 \mathrm{rpm}$ for $20 \mathrm{~min}$ at $4^{\circ} \mathrm{C}$. The supernatant was collected and the total protein was determined using the Bradford method. ${ }^{15}$ The tissue levels of TNF- $\alpha$ were measured by a commercially available rat ELISA kit (eBioscience, Inc., San Diego, USA), according to the instructions of the manufacturer and expressed as ng/g protein.

\section{Measurement of the tissue protein levels}

The total protein concentration in the tissue samples was determined according to Bradford's method using bovine serum albumin as a standard. ${ }^{15}$

\section{Histopathological evaluation}

Formalin-fixed hippocampus sections $(5 \mu \mathrm{m})$ were stained with Cresyl violet stain. All sections were examined with an Olympus C-5050 digital camera at an Olympus BX51 microscope. Cresyl violet staining to quantify the number of surviving neurons in the CA1 and CA3 regions of the hippocampus were performed in 6 sections per studied group using an image analysis system (ImagePro Express 1.4.5, Media Cybernetics, Inc., Rockville, USA).

\section{Statistical analysis}

Statistical evaluation was performed using SPSS v. 15.0 for Windows. The groups of parametric variables were compared using the Student's t test and analysis of variance. The groups of nonparametric variables were compared using the Mann-Whitney $U$ test. In addition, the Shapiro-Wilk test was used for parametric and nonparametric differentiation. The results are presented as mean \pm SEM. A p value of $<0.05$ was accepted as statistically significant.

\section{Results}

The latency time, brain TNF- $\alpha$ level and ChAT activity, and the number of neurons in the CA1 and CA3 hippocampal regions in the animals are shown in Table 1. Analysis of the data showed a significantly shorter latency time, higher brain TNF- $\alpha$ level, and lower ChAT activity and number of neurons in the CA1 and CA3 hippocampal regions in the ICV-STZ and saline group compared to the sham group and control group. On the other hand, latency time was longer, brain TNF- $\alpha$ level was lower, and brain ChAT activity and the number of neurons in the CA1 and CA3 hippocampal regions were higher in the ICV-STZ and rhEPO group compared to the ICV-STZ and saline group.

The Cresyl violet-stained histological preparations are shown in Fig. 1. The cell layer was thinner and the number of neurons was lower in CA1 and CA3 hippocampal regions in the ICV-STZ and saline group compared to the ICV-STZ and rhEPO group. The histopathological findings in the ICV-STZ and EPO-treated animals were similar to that of the sham and control rats.

\section{Discussion}

ICV-STZ injection is an experimental sporadic-AD animal model that has been frequently used recently. STZ is a diabetogenic substance that is particularly toxic to the pancreatic B-cells and insulin receptors in the brain of 
Table 1. Latency time, brain TNF-a level and ChAT activity, and the number of neurons in hippocampal CA1 and CA3 regions of groups

\begin{tabular}{|l|c|c|c|} 
& Control group & Sham group & ICV-STZ and saline group \\
\hline Latency time $(\mathrm{s})$ & $214.66 \pm 46.5$ & $249.1 \pm 24.9$ & $49.5 \pm 15.05^{* *}$ \\
ChAT (U/g protein) & $142.76 \pm 8.91$ & $157.8 \pm 10.6$ & $93.15 \pm 14.6^{* *}$ \\
Brain TNF-a level (ng/g protein) & $0.31 \pm 0.04$ & $0.40 \pm 0.06$ & $195.83 \pm 43.15^{\#}$ \\
Number of CA1 neurons & $66.25 \pm 3.22$ & $62.36 \pm 3.56$ & $124.7 \pm 8.9^{*}$ \\
Number of CA3 neurons & $61.63 \pm 3.89$ & $59.26 \pm 3.32$ & $1.58 \pm 0.20^{*}$ \\
\hline
\end{tabular}

Data expressed as mean \pm SEM. ChAT: choline acetyltransferase, EPO: erythropoietin, TNF-a: tumor necrosis factor-alpha; ** $\mathrm{p}<0.01$, ICV-STZ and Saline Group vs. Sham Group or Control Group; * $p<0.05$, ICV-STZ and Saline Group vs ICV-STZ and rhEPO Group; ${ }^{\text {p }}<0.01$, ICV-STZ and Saline Group vs ICV-STZ and rhEPO Group.

mammals. ${ }^{16}$ ICV administration of STZ desensitizes neuronal insulin receptors, reduces the activity of glycolytic enzymes and causes severe abnormalities in metabolic pathways, which are under the control of the insulin signaling cascade in the rat brain. ${ }^{17}$ Accordingly, ICV-STZ probably induces cognitive impairments via disturbances in glucose/energy metabolism by inhibiting the insulin receptor system and induction of oxidative stress by inhibiting ATP synthesis and acetyl-coenzyme A and thus acetylcholine (ACh) synthesis. On the other hand, ICVSTZ-induced oxidative damage increases the inflammatory cytokines which leads to mitochondrial dysfunction and increases the risk of cell apoptosis in the brain, particularly in the hippocampus and cortex. Otherwise, disturbances in insulin signaling may also augment tau phosphorylation and potentiates $A \beta$ toxicity, which would lead to the neuronal degeneration and cognitive dysfunction that occurs with AD. ${ }^{8,13,18}$

Learning and memory impairment is the first and the most characteristic symptom in AD. ${ }^{1}$ STZ-ICV-treated rats consistently demonstrate deficits in learning and memory, regardless of age. Long-term cognitive deficits have been observed as early as 2 weeks after single or double ICV-STZ (3 mg/kg) injections and were maintained at least 12-14 weeks. ${ }^{12,13}$ Accordingly, we found significant memory deficits on the PAL test in the ICV-STZ (single, $3 \mathrm{mg} / \mathrm{kg}$ ) group 2 weeks after STZ administration. RhEPO treatment significantly prevented memory impairment as indicated by an improvement in performance on the PAL. This result is in accordance with previous studies that have demonstrated that rhEPO improves memory function and cognitive functioning in animal models of acute and chronic neurodegenerative disorders and neuropsychiatric illness, and in patients with cognitive decline and even in healthy volunteers. ${ }^{7,9}$ Repeated rhEPO treatment also increased hippocampal memory in healthy mice. ${ }^{19}$ Indeed, in clinical studies, chronic treatment with rhEPO improved neurocognitive dysfunction in patients with schizophrenia ${ }^{20}$ and multiple sclerosis ${ }^{21}$ as well as with prematurity ${ }^{22}$ and coronary artery surgery. ${ }^{23}$

The degeneration of basal forebrain cholinergic neurons and the associated defects of central cholinergic neurotransmission into the cerebral cortex and other areas have been suggested as the principal cause of cognitive dysfunction in $\mathrm{AD} .{ }^{24} \mathrm{ChAT}$ is one of the specific cholinergic marker proteins for the function of cholinergic neurons, which are responsible for ACh synthesis in these neurons. Their levels dramatically decrease in the hippocampus and frontal cortex of brain slices from AD patients. Cholinergic deficits have been also found in ICV-STZ-induced animals as a decrease in ChAT activity and an increase in acetylcholinesterase activity in the brain, particularly the hippocampus. ${ }^{13}$ In this study, ChAT activity was significantly lower in the brain tissue of $\mathrm{ICV}-\mathrm{STZ}$ + saline treated rats, and treatment with rhEPO maintained ChAT expression. This finding is in accordance with previous studies reporting increased ChAT activity induced by rhEPO in STZ- and lipopolysaccharide-induced sporadic-AD animal models..$^{25,26}$

Neuroinflammation is also an important component in the pathogenesis and progression of AD. TNF- $\alpha$ is the major cytokine synthesized by activated microglia and neurons and initiating the inflammatory cascade. It is expressed at very low levels in healthy adult brains, but examinations of postmortem AD brains has revealed that increased TNF- $\alpha$ co-localizes with A $\beta$ plaques, and TNF- $\alpha$ is up-regulated in both CSF and serum, and correlates with the disease severity. ${ }^{2}$ In AD brains, TNF- $\alpha$ can exacerbate phospho-tau pathologies and contribute to amyloidogenesis via beta-secretase regulation; cause insulin resistance and $\mathrm{BBB}$ deterioration; induce chronic inflammation, glutamate excitotoxicity, oxidative stress and mitochondrial dysfunction; impair neurogenesis, synaptic transmission and plasticity that may promote synaptic loss and cognitive dysfunction and, eventually, neuronal death. ${ }^{2,16}$ Because of all these detrimental effects of TNF- $\alpha$ as well as the beneficial effects of anti-TNF medications in $\mathrm{AD}$, the TNF signal has been suggested as a novel therapeutic target for AD. Therefore, in a valid rat model of AD induced by ICV-STZ, which has been shown to be characterized by increased proinflammatory cytokines including TNF- $\alpha$ and IL-1 $\beta^{2,13}$, we have evaluated the possible effects of rhEPO on TNF- $\alpha$ levels. We found increased an TNF- $\alpha$ level in ICV-STZ-infused rats with 


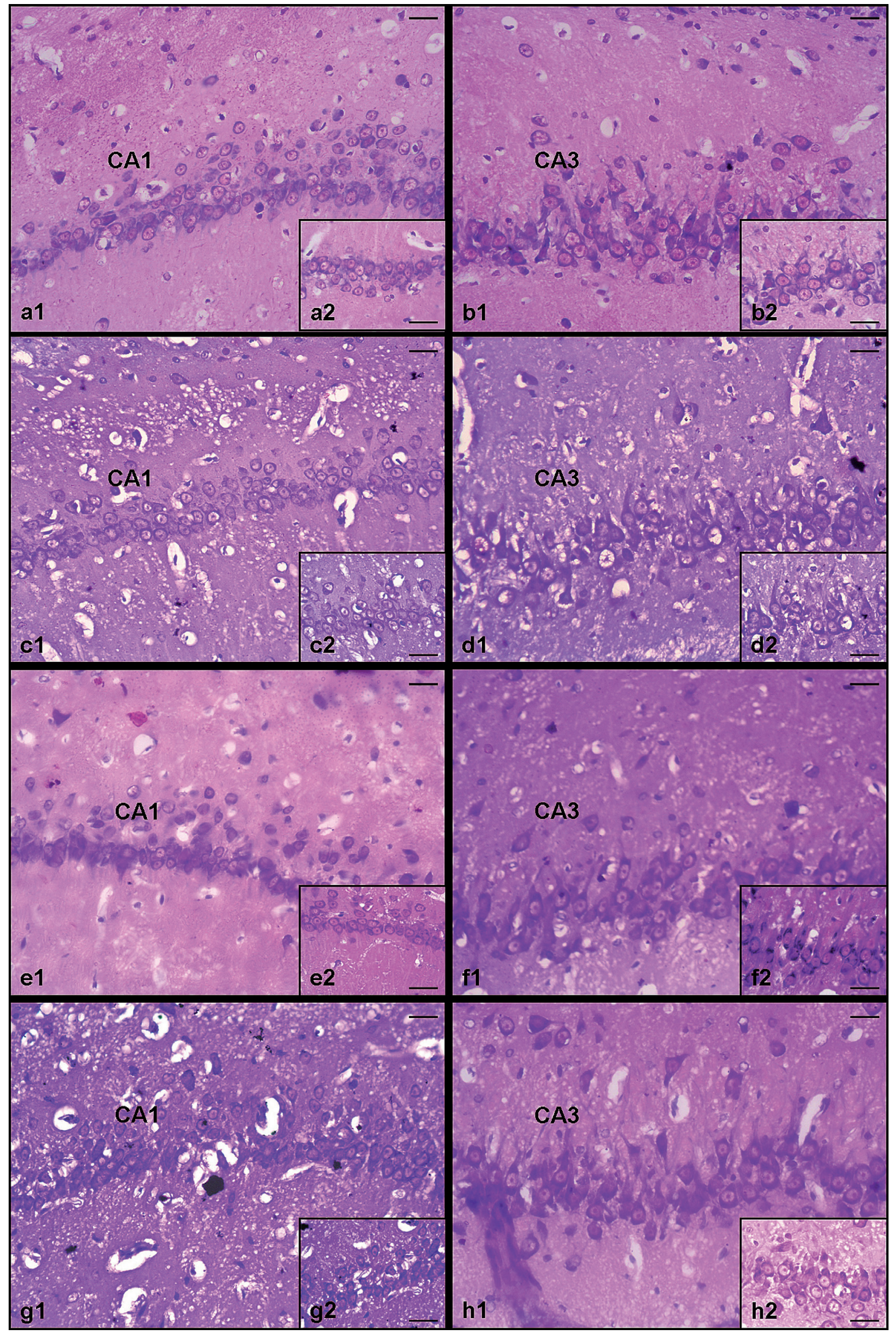

Fig. 1. CA1 and CA3 hippocampal regions were stained with Cresyl violet stain ( $x 40$ and $\times 100$ magnification). $a 1$ and a2 : Control Group CA1, b1 and b2: Control Group CA3, C1 and c2: Sham Group CA1, d1 and d2: Sham Group CA3, e1 and e2: ICV-STZ and Saline Group CA1, f1 and f2: ICV-STZ and Saline Group CA3, g1 anf g2: ICV-STZ and EPO Group CA1, h1 and h2: ICV-STZ and EPO Group CA3 
saline treatment vs. a significantly decreased TNF- $\alpha$ level in the rhEPO-treated group. The results of the present study is in accordance with previous in vivo experimental studies, reporting that rhEPO decreases the expression of proinflammatory cytokines including TNF- $\alpha$, IL- 6 and monocyte chemoattractant- 1 , the expression of chemokines, microglial activation, and cerebral leukocyte influx in the central nervous system. ${ }^{27-29}$

The hippocampus has a key role in learning and memory formation and is one of the first structures of the brain to undergo damage in AD. ${ }^{8}$ In the present study, we showed marked neuronal loss in the $\mathrm{C} 1$ and $\mathrm{C} 3$ regions of the hippocampus in ICV-STZ-induced rats. However, rhEPO significantly prevented neuronal loss in these regions. These results are in accordance with previous in vitro studies that showed rhEPO protects cultured hippocampal neurons from the $A \beta$, hypoxia, glucose/serum deprivation, TNF- $\alpha$, trauma, nitric oxide, and glutamate induced apopitosis that are involved in the pathogenesis of AD. ${ }^{11}$ Also, in vivo studies have showed that a learning disability induced by ischemia, trauma or hippocampal lesions can be attenuated by local/systemic administration of rhEPO or its variants. ${ }^{9}$ There are a limited number of studies about the effects of EPO on AD. RhEPO has been tested in the ICV-STZ and i.p.-scopolamine models with doses of 500 and 1,000 IU/kg i.p., and significantly reversed hippocampal memory deficits along with attenuation of oxidative stress and restoration of AChE activity in mouse brains. ${ }^{26}$ In aged (Tg2576) mice, rhEPO (5000 IU/ $\mathrm{kg} /$ day, i.p. for 5 days) improved the contextual memory with reducing $A \beta$-induced endothelial dysfunction and the amount of amyloid plaque in the brain. ${ }^{5}$ Furthermore, in ICV-STZ-induced rats, rhEPO treatment $(5000 \mathrm{IU} / \mathrm{kg} /$ day, i.p. for 2 weeks) not only reversed the spatial learning and memory deficits ${ }^{8}$, but also improved neuronal proliferation in the dentate gyrus. ${ }^{19}$ In a ICV-A $\beta_{25-35}$ mouse model, rhEPO prevented memory impairment, neuronal loss of the hippocampal CA1 region, induction of lipid peroxidation and TNF- $\alpha$ and IL- $1 \beta$ production. ${ }^{27}$ Interestingly, EPO promotes mesenchymal stem cells to differentiate into cholinergic neurons, resulting in increased ChAT activity and ACh level in lipopolysaccharide-treated mice. $^{25}$

Both EPO and its receptor (EPOR) are expressed by glial cells, neuronal pregenitor cells, neurons and cerebrovascular endothelium in rodent and human brains, and show high immunopositivity in the frontal cortex and hippocampus. ${ }^{30}$ EPOR is upregulated in the brain upon injury or neurodegenerative conditions, such as in the temporal cortex and hippocampus of patients with mild cognitive impairment or $\mathrm{AD}$, which may be because of the increased cell requirements for this hormone. This data suggests that EPO signaling plays an important role in hippocampal functioning and neuroprotection. ${ }^{3}$ Although the exact neuroprotective and neuroregenerative mechanisms of erythropoietin are not clear, possible mechanisms are antiapoptotic, antioxidative, antiexcitotoxic, antiinflammatory and neurotrophic activity; stimulation of angiogenesis, oligodendrogenesis and axonal remodeling; protection of BBB integrity and cerebral blood flow; and modulation of intracellular calcium and nitric oxide by binding with EPOR within the brain. ${ }^{6-10}$ EPO can induce neurogenesis in the hippocampus and therefore improves hippocampus-dependent memory. EPO enhances glucose transporters, glycolytic enzyme activities and growth factor production. It also increases gene expression of neurotrophic factors such as the brain-derived neurotrophic factor, insulin growth factor 1 and certain associated proteins in the hippocampus. ${ }^{8}$ In addition, EPO-enhanced long-term potentiation and altered short-term synaptic plasticity and synaptic transmission, shifting the balance of excitatory and inhibitory activity. ${ }^{19}$

This study only evaluates the acute effects of rhEPO for 20 days after ICV-STZ injection. However, this period of time is shorter than the previously-reported time (almost 6-9 months) to develop almost complete AD pathology like $\mathrm{A} \beta$ plaques and neurofibrillary tangles. ${ }^{13}$ Thus, both the long term effect of ICV-STZ on rat brains and the effects of rhEPO on ICV-STZ-induced alterations in rat brains is lacking.

In this study, we observed that rhEPO treatment significantly prevented the ICV-STZ-induced memory deficit via attenuating hippocampal neuronal loss, neuroinflammation and cholinergic deficit in rats. This result suggests that rhEPO may be beneficial for treating AD.

\section{References}

1. Grand JH, Caspar S, Macdonald SW. Clinical features and multidisciplinary approaches to dementia care. J Multidiscip Healthc. 2011;4:125-147.

2. Montgomery SL, Bowers WJ. Tumor necrosis factor-alpha and the roles it plays in homeostatic and degenerative processes within the central nervous system. J Neuroimmune Pharmacol. 2012;7:42-49.

3. Assaraf MI, Diaz Z, Liberman A, et al. Brain erythropoietin receptor expression in Alzheimer disease and mild cognitive impairment. J Neuropathol Exp Neurol. 2007;66:389-398.

4. Khairallah MI, Kassem LA. Alzheimer's disease: Current status of etiopathogenesis and therapeutic strategies. PakJ Biol Sci. 2011;14: 257-272.

5. Lee ST, Chu K, Park JE, Jung KH, Jeon D, Lim JY, Kim M, JK. Erythropoietin improves memory function with reducing endothelial dysfunction and amyloid-beta burden in Alzheimer's disease models. J Neurochem. 2012;120:115-124.

6. Byts N, Sirén AL. Erythropoietin: A multimodal neuroprotective agent. Exp Trans/ Stroke Med. 2009;1:4.

7. Miskowiak KW, Vinberg M, Harmer CJ, Ehrenreich H, Kessing LV. Erythropoietin: A candidate treatment for mood symptoms and memory dysfunction in depression. Psychopharmacology (Berl). 2012;219:687-698.

8. Hamidi G, Arabpour Z, Shabrang M, Rashidi B, et al. Erythropoietin improves spatial learning and memory in streptozotocin model of dementia. Pathophysiology. 2013;20:153-158.

9. Sargin D, Friedrichs H, El-Kordi A, Ehrenreich H. Erythropoietin as neuroprotective and neuroregenerative treatment strategy: comprehensive overview of 12 years of preclinical and clinical research. Best Pract Res Clin Anaesthesiol. 2010;24:573-594.

10. Kowalczyk M, Banach M, Mikhailidis DP, Rysz J. Erythropoietin update 2011. Med Sci Monit. 2011;17:240-247. 
11. Subirós N, Del Barco DG, Coro-Antich RM. Erythropoietin: Still on the neuroprotection road. Ther Adv Neurol Disord. 2012;5:161-173.

12. Mehla J, Pahuja M, Gupta YK. Streptozotocin-induced sporadic Alzheimer's disease: selection of appropriate dose. J Alzheimers Dis. 2013;33:17-21.

13. Salkovic-Petrisic M, Knezovic A, Hoyer S, Riederer P. What have we learned from the streptozotocin-induced animal model of sporadic Alzheimer's disease, about the therapeutic strategies in Alzheimer's research. J Neural Transm. 2013;120:233-252.

14. Paxinos G, Watson C. The Rat Brain in Stereotaxic Coordinates, Spiral Bound, Academic Press, New York 1998, $4^{\text {th }}$ ed.

15. Bradford MM. A rapid and sensitive method for the quantitation of microgram quantities of protein utilizing the principle of protein-dye binding. Anal Biochem. 1976;72:248-254.

16. Clark I, Atwood C, Bowen R, Paz-Filho G, Vissel B. Tumor necrosis factor-induced cerebral insulin resistance in Alzheimer's disease links numerous treatment rationales. Pharmacol Rev. 2012;64:1004-1026.

17. Pinton S, da Rocha JT, Gai BM, Nogueira CW. Sporadic dementia of Alzheimer's type induced by streptozotocin promotes anxiogenic behavior in mice. Behav Brain Res. 2011;223:1-6.

18. Arabpoor Z, Hamidi G, Rashidi B, et al. Erythropoietin improves neuronal proliferation in dentate gyrus of hippocampal formation in an animal model of Alzheimer's disease. Adv Biomed Res. 2012;1:50.

19. Adamcio B, Sargin D, Stradomska A, Medrihan L, Gertler C, Theis F. Erythropoietin enhances hippocampal long-term potentiation and memory. BMC Biol. 2008;6:37.

20. Wüstenberg T, Begemann M, Bartels $C$, et al. Recombinant human erythropoietin delays loss of gray matter in chronic schizophrenia. Mol Psychiatry. 2011;16:26-36.

21. Ehrenreich $\mathrm{H}$, Fischer $\mathrm{B}$, Norra $\mathrm{C}$, et al. Exploring recombinant human erythropoietin in chronic progressive multiple sclerosis. Brain. 2007;130:2577-2588.
22. Bierer R, Peceny MC, Hartenberger $\mathrm{CH}$, Ohls RK. Erythropoietin concentrations and neurodevelopmental outcome in preterm infants. Pediatrics. 2006;118:635-640.

23. Haljan G, Maitland A, Buchan A, et al. The erythropoietin neuroprotective effect: assessment in CABG surgery (TENPEAKS): A randomized, double-blind, placebo controlled, proof-of-concept clinical trial. Stroke. 2009;40:2769-2775.

24. Francis PT, Palmer AM, Snape M, Wilcock GK. The cholinergic hypothesis of Alzheimer's disease: A review of progress. J Neurol Neurosurg Psychiatry. 1999;66:137-147.

25. Khairallah MI, Kassem LA, Yassin NA, et al. The hematopoietic growth factor "erythropoietin" enhances the therapeutic effect of mesenchymal stem cells in Alzheimer's disease. PakJ Biol Sci. 2014; 17:9-21.

26. Kumar R, Jaggi AS, Singh N. Effects of erythropoietin on memory deficits and brain oxidative stress in the mouse models of dementia. Korean J Physiol Pharmacol. 2010;14:345-352.

27. Maurice $\mathrm{T}$, Mustafa $\mathrm{MH}$, Desrumaux $\mathrm{C}$, et al. Intranasal formulation of erythropoietin (EPO) showed potent protective activity against amyloid toxicity in the $A \beta_{25-35}$ non-transgenic mouse model of Alzheimer's disease. J Psychopharmacol. 2013;27:1044-1057.

28. Noh MY, Cho KA, Kim H, Kim SM, Kim SH. Erythropoietin modulates the immune-inflammatory response of a SOD1(G93A) transgenic mouse model of amyotrophic lateral sclerosis (ALS). Neurosci Lett. 2014;574:53-58.

29. Villa P, Bigini P, Mennini, T et al. Erythropoietin selectively attenuates cytokine production and inflammation in cerebral ischemia by targeting neuronal apoptosis. J Exp Med. 2003;198:971-975.

30. Diederich K, Schäbitz WR, Minnerup J. Seeing old friends from a different angle: novel properties of hematopoietic growth factors in the healthy and diseased brain. Hippocampus. 2012;22:10511057. 